\title{
Failure of both suction catheter passage and bronchoscopy to diagnose an obstructing tracheal mucus plug
}

\author{
J. Adam Law, MD · Carlo Mariotti, MD • \\ Tim Mullen, MD
}

Received: 9 May 2012/Accepted: 15 May 2012/Published online: 30 May 2012

(c) Canadian Anesthesiologists' Society 2012

\section{To the Editor,}

The patient's spouse gave informed consent for publication of this report. A 55-yr-old male sustained burns in a welding accident extending caudad from shoulder level over $65 \%$ of his body surface area. In the intervening two months, he required multiple surgeries, including debridement and skin grafting, a laparotomy for abdominal compartment syndrome, and a tracheostomy. Presenting on this occasion for further skin grafting, he was breathing easily and spontaneously through a size 8 fenestrated Shiley ${ }^{\mathrm{TM}}$ tracheostomy cannula (Covidien, Mansfield, MA, USA) capped with a decannulation plug. In the operating room, the fenestrated inner cannula was exchanged for a non-fenestrated tube. Shortly after this change, the patient indicated some dyspnea. A suction catheter was introduced and moderate amounts of sputum were obtained, after which the patient signalled at least partial relief. Following application of routine monitors, anesthesia was induced using sevoflurane in oxygen through a circle system connected to the tracheostomy tube, supplemented with midazolam $2 \mathrm{mg}$ and propofol $30 \mathrm{mg} i v$.

After induction, it was almost impossible to ventilate the patient's lungs. We detected a markedly prolonged expiratory phase and made a working diagnosis of severe bronchospasm although minimal rhonchi were present. We administered rocuronium $50 \mathrm{mg} i \mathrm{v}$, increased the inspired sevoflurane concentration to $4 \%$, and administered salbutamol, magnesium sulphate, and small doses (50-100 $\mu \mathrm{g})$ of intravenous epinephrine. Although the oxygen saturation remained $>90 \%$, little improvement was achieved in

J. A. Law, MD $(\varangle) \cdot$ C. Mariotti, MD · T. Mullen, MD Dalhousie University/QEII Health Sciences Centre, Halifax, NS, Canada

e-mail: jlaw@dal.ca response to these measures. An 18-Fr suction catheter was introduced on at least three further occasions. Each time, a scant amount of sputum was obtained, but no improvement in ventilation resulted. We twice passed a fibreoptic bronchoscope, but saw no obvious impediment to ventilation, and the tracheobronchial tree looked normal. Interestingly, when the tracheostomy tube was disconnected from the circuit for suctioning or bronchoscopy, we observed the patient's chest gradually fall with loud yet slow gas egress from the tracheostomy cannula, suggesting gas escaping under conditions of high intrathoracic pressure. Following each such thoracic decompression, ventilation was easier for the next two or three positive pressure inspirations. Eventually, an inspiratory:expiratory (i:e) ratio of about 1:10 was observed to allow sufficient time for expiration, such that ventilation was now possible, albeit with ongoing high airway pressures. To rule out a circuit or machine problem, ventilation was assessed with a manual resuscitator, which confirmed that the high airway pressures originated from either the tracheostomy cannula or the patient. A portable chest radiograph showed only bilateral lung hyperinflation. Although substantially hypercarbic $\left(\mathrm{PetCO}_{2}\right.$ up to $93 \mathrm{mmHg}$ ), fortunately, the patient suffered no cardiovascular instability.

Ultimately, a change of tracheostomy was planned given the poor response to treatment for bronchospasm, ongoing problems with positive pressure ventilation, evidence of air trapping, and the initial history of dyspnea with tracheostomy manipulation. Repeat bronchoscopy was performed by a different clinician using a videobronchoscope. The visual field was briefly obscured as the videobronchoscope was advanced beyond the distal end of the tracheostomy cannula, after which a clear view of the trachea was observed. At this point, the possibility of cuff herniation was considered. Direct laryngoscopy prior to tracheostomy 
tube exchange revealed a Cormack-Lehane Grade 2 view. The patient's trachea was decannulated, and a new size 8 Shiley tracheostomy tube was placed uneventfully. Ventilation promptly became non-problematic. The issue became immediately evident from the removed tracheostomy tube, as a thick mucus plug was occluding the end of the cannula (Figure). The plug had allowed inspiration with positive pressure ventilation, but acted as a ball valve in preventing all but minimal gas egress. The plug did not impede passage of a suction catheter and had not been appreciated by the initial bronchoscopist who later reported that he had probably passed the scope through and beyond the distal end of the tracheostomy tube, and likely through the obstructing plug, before looking through the bronchoscope's eyepiece.

Although mucus plugging is not an uncommon problem, there are few published reports detailing these challenges. This particular experience underscores that successful and easy passage of a suction catheter does not rule out a partially obstructed tube, nor does passage of a bronchoscope indicate patency without obtaining and interpreting views correctly. The case lends credence to the often quoted phrase, "if in doubt, take it out", and finally to the importance of modifying the differential diagnosis based

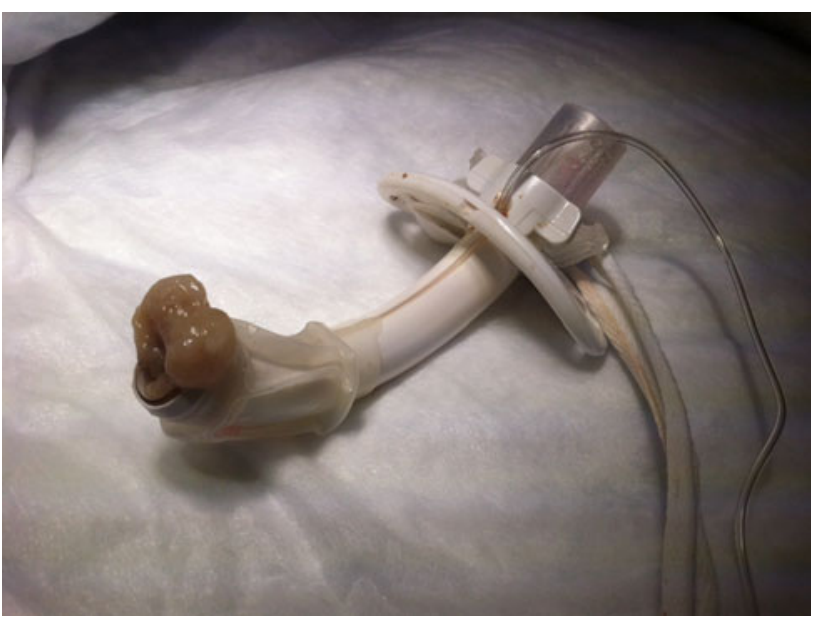

Figure Obstructing mucus plug on the end of the removed Shiley ${ }^{\mathrm{TM}}$ tracheostomy tube

on response/non-response to specific therapeutic airway interventions.

Competing interests This work was unfunded. None of the authors has any commercial or non-commercial affiliations that would constitute a conflict of interest with this work or any other relevant associations. 